\title{
VEGF promotes endothelial progenitor cell differentiation and vascular repair through connexin 43
}

\author{
Lufeng Li ${ }^{1}$, Huanyun Liu ${ }^{1,2}$, Chunxin $\mathrm{Xu}^{1}$, Mengyang Deng ${ }^{1}$, Mingbao Song ${ }^{1}$, Xuejun Yu' ${ }^{1}$, Shangcheng $\mathrm{Xu}^{3}$
} and Xiaohui Zhao ${ }^{{ }^{*}}$

\begin{abstract}
Background: Endothelial progenitor cell (EPC) differentiation is considered crucial for vascular repair. Vascular endothelial growth factor (VEGF) induces EPC differentiation, but the underlying mechanism of this phenomenon remains unclear. Connexin 43 (Cx43) is reported to be involved in the regulation of stem cell differentiation. Therefore, we sought to determine whether Cx43 is involved in VEGF-induced EPC differentiation and vascular repair.

Methods: Rat spleen-derived EPCs were cultured and treated with various concentrations of VEGF $(0,10$, or $50 \mathrm{ng} / \mathrm{mL})$, and the relationship between EPC differentiation and Cx43 expression was evaluated. Thereafter, fluorescence redistribution after photobleaching was performed to assess the relationship between adjacent EPC differentiation and Cx43-induced gap junction intercellular communication (GJIC). After carotid artery injury, EPCs pretreated with VEGF were injected into the tail veins, and the effects of $C \times 43$ on vascular repair were evaluated.

Results: EPCs cultured with VEGF exhibited accelerated differentiation and increased expression of Cx43. However, inhibition of Cx43 expression using short interfering RNA (siRNA) attenuated EPC GJIC and consequent EPC differentiation. VEGF-pretreated EPC transplantation promoted EPC homing and reendothelialization, and inhibited neointimal formation. These effects were attenuated by siRNA inhibition of Cx43.

Conclusions: Our results from in vivo and in vitro experiments indicated that VEGF promotes EPC differentiation and vascular repair through $\mathrm{C} \times 43$.
\end{abstract}

Keywords: Endothelial progenitor cell, Vascular endothelial growth factor, Connexin 43, Differentiation, Vascular repair

\section{Background}

Vascular endothelial injury has been identified as a trigger of and critical contributor to atherosclerosis [1, 2]. Previous studies have suggested that endothelial progenitor cells (EPCs) derived from the bone marrow or spleen have the potential to be incorporated into injured areas and differentiate into endothelial cells (ECs), thereby contributing to the improvement of endothelial function [3, 4]. Vascular endothelial growth factor (VEGF) plays an important role in EPC differentiation and vascular repair $[5,6]$, which involves multiple signaling pathways including mitogen-activated protein kinase/

\footnotetext{
* Correspondence: doctorzhaoxiaohui@yahoo.com

${ }^{1}$ Institute of Cardiovascular Research, Xinqiao Hospital, Third Military Medical

University, Chongqing 400037, China

Full list of author information is available at the end of the article
}

extracellular signal-related kinase (MAPK/ERK) [7, 8]. However, to date, the mechanisms underlying VEGFmediated EPC differentiation remain unclear.

Gap junctions (GJs), which are composed of connexins, are an important contributor to direct intercellular communications, mediating the exchange of ions, metabolites, and secondary messenger molecules between neighboring cells [9]. Among the 21 members of the connexin family, connexin $43(\mathrm{Cx} 43)$ is reported to be a major GJ protein in multiple cells and is essential for the regulation of stem cell differentiation [10-12]. We found that blocking intercellular communication via $\mathrm{Cx} 43$ hemichannels reduces neointimal formation after vascular injury by inhibiting the proliferation and phenotypic modulation of smooth 
muscle cells [13]. Furthermore, it has been reported that blocking Cx43 expression inhibits GJ-mediated signal transfer between adjacent cells, thereby impairing EPC proliferation, migration, and angiogenesis; this can be visualized by observing the inhibition of fluorescence flow between cells $[14,15]$. However, whether $\mathrm{Cx} 43$ participates in VEGF-induced EPC differentiation remains unclear. Therefore, in the present study, we investigated the effects of Cx43-mediated GJ function on EPC differentiation and vascular repair.

\section{Methods}

\section{EPC culture and characterization}

EPC culture and characterization were performed as previously described [16, 17]. Briefly, mononuclear cells (MNCs) were isolated from the spleens of SpragueDawley rats (male, 150-180 g; Chongqing, China) by density gradient centrifugation with Ficoll separation solution. After rinsing three times with phosphate-buffered saline (PBS; Sigma-Aldrich, St Louis, MO, USA), MNCs were seeded on gelatin-coated cell culture plates $\left(1 \times 10^{6}\right.$ cells $\left./ \mathrm{cm}^{2}\right)$ and maintained in Dulbecco's modified Eagle's medium (DMEM; Gibco, Waltham, MA, USA) supplemented with $20 \%$ fetal calf serum in a $5 \% \mathrm{CO}_{2}$ incubator at $37{ }^{\circ} \mathrm{C}$. The medium was changed every 3 days, and only attached cells were used in subsequent experiments.

For characterization, cells were incubated with $2.4 \mathrm{ng} / \mathrm{mL}$ DiI-labeled acetylated low-density lipoprotein (DiI-ac-LDL; Invitrogen, Carlsbad, CA, USA) for $1 \mathrm{~h}$ at $37{ }^{\circ} \mathrm{C}$ and fixed in $2 \%$ paraformaldehyde (PFA; CellChip Biotechnology, Shenzhen, China) for $15 \mathrm{~min}$. After washing with PBS, cells were stained with $10 \mathrm{mg} / \mathrm{mL}$ fluorescein isothiocyanate-labeled Ulex europaeus agglutinin I (FITC-UEA-I; Sigma-Aldrich) for $1 \mathrm{~h}$. The samples were rinsed three times with PBS and evaluated using laser scanning confocal microscopy (LSCM; Leica, Wetzlar, Germany). Cells positive for both DiI-ac-LDL and FITCUEA-I were regarded as differentiating EPCs. Additionally, EPC phenotypes were determined by fluorescenceactivated cell sorting (FACS) with two antibodies: FITCconjugated anti-CD34 (Abcam, Cambridge, MA, USA) and phycoerythrin (PE)-conjugated anti-VEGF receptor 2 (VEGFR2; Abcam).

\section{Immunofluorescence analysis}

To evaluate the expression of Cx43, EPCs were fixed in 4\% PFA for $15 \mathrm{~min}$ and washed three times with PBS. Then, cells were permeabilized with $0.1 \%$ Triton X-100 for $15 \mathrm{~min}$ and treated with serum sealing fluid for $30 \mathrm{~min}$. EPCs were first incubated with anti-Cx43 primary antibody (Abcam; 1:500), and then with FITClabeled secondary antibody (Beyotime, Shanghai, China). After washing with PBS, EPCs were incubated with 4',6- diamidino-2-phenylindole (DAPI) staining solution. Images were obtained using LSCM (Leica).

\section{Semi-quantitative reverse transcriptase (RT)-PCR}

After incubation with $\operatorname{VEGF}(0,10$, or $50 \mathrm{ng} / \mathrm{mL})$ for $24 \mathrm{~h}$, EPCs were harvested. Total RNA was extracted with RNAiso Plus (Takara, Tokyo, Japan) according to the manufacturer's instructions. DNA was obtained through RT-PCR using a PrimeScript RT reagent kit and Max PCR Master Mix (Takara) with total RNA as the template. The Cx43-specific primer (163 bp) was synthesized by Fulengen (Guangzhou, China). $\beta$-actin was used as a control, and the primer sequences (Takara) were $5^{\prime}$ CCGTAAAGACCTCTATGCCAAC-3' (sense) and 5'ACTCATCGTACTCCTGCTTGCT-3' (antisense), for a product length of $227 \mathrm{bp}$.

\section{Western blot analysis}

After incubation with VEGF $(0,10$, or $50 \mathrm{ng} / \mathrm{mL})$ for $24 \mathrm{~h}$, EPCs were harvested. Cells were lysed with radioimmunoprecipitation assay (RIPA) buffer (Beyotime) containing the protease inhibitor phenylmethane sulfonyl fluoride (PMSF; 100:1). The lysates were centrifuged at 12,000 $\times g$ and $4{ }^{\circ} \mathrm{C}$ for $20 \mathrm{~min}$, and the protein concentration of the supernatant was determined using the bicinchoninic acid assay kit (Beyotime). Identical concentrations of protein were subjected to SDS-PAGE and transferred to polyvinylidene fluoride (PVDF) membranes (EMD Millipore, Billerica, MA, USA). After treatment with $5 \%$ bovine serum albumin (BSA) blocking reagent (Solarbio, Beijing, China) for $60 \mathrm{~min}$ at $25{ }^{\circ} \mathrm{C}$, membranes were further incubated with anti-Cx43, anti-CD31, anti-von Willebrand factor (vWF), anti-glyceraldehyde 3phosphate dehydrogenase (GAPDH), or anti- $\beta$-actin (Abcam). After washing with PBS three times, membranes were probed with corresponding horseradish peroxidase-coupled secondary antibodies (Beyotime). Protein bands were visualized with an enhanced chemiluminescence (ECL) detection system (Pierce, Waltham, MA, USA) and quantified using a gel image analysis system (Bio-Rad, Hercules, CA, USA).

\section{RNA interference}

Cells were transfected with $8 \mu \mathrm{l} \mathrm{Cx} 43$ short interfering RNA (siRNA; siCx43; SC6008, Santa Cruz Biotechnology, Dallas, TX, USA) for $6 \mathrm{~h}$ at $37^{\circ} \mathrm{C}$ in a $5 \% \mathrm{CO}_{2}$ incubator. Then, cells were incubated with DMEM containing 20\% fetal calf serum for an additional $48 \mathrm{~h}$. For this experiment, transfection reagent (SC29528, Santa Cruz Biotechnology), transfection medium (SC36868, Santa Cruz Biotechnology), and FITC-labeled control siRNA (SC37007, Santa Cruz Biotechnology) were used. The proteins generated were quantified via western blot. 
Fluorescence redistribution after photobleaching (FRAP) FRAP experiments were performed as previously described $[13,18]$. The control group was treated with saline for $24 \mathrm{~h}$, and the experimental group was treated with $50 \mathrm{ng} / \mathrm{mL}$ VEGF for $24 \mathrm{~h}$. The EPC + VEGF + siCx43 group was treated with $8 \mu \mathrm{Cx} 43$ siRNA for $6 \mathrm{~h}$ following VEGF treatment.

EPCs under different treatments were loaded with the $\mathrm{Ca}^{2+}$-insensitive dye 6-carboxy-fluorescein diacetate $\left(10 \mu \mathrm{g} / \mathrm{mL}\right.$; Sigma-Aldrich) for $10 \mathrm{~min}$ at $37{ }^{\circ} \mathrm{C}$. Then, FRAP results were measured using a confocal microscope (Leica). The dye was excited at $488 \mathrm{~nm}$, and its emission recorded at $570 \mathrm{~nm}$. Before bleaching, polygons were drawn around the cells chosen for bleaching, and three pre-bleached images were scanned with a weaker laser. The cells chosen for bleaching were then exposed to 50 scans with a laser at $95 \%$ intensity, and the recovery of fluorescence in the bleached cells was measured every $15 \mathrm{~s}$ over $3 \mathrm{~min}$. After correction for background bleaching, the recovery of fluorescence in the bleached cells at $3 \mathrm{~min}$ was compared with that of the pre-bleached scans, and the recovery percentage was calculated.

\section{Differentiation assay}

CD31 and vWF are two important surface antigens found on ECs. Therefore, we detected the expression of CD31 and vWF using a western blot to confirm the differentiation of EPCs into ECs.

\section{Carotid artery injury and identification}

Rats were randomly assigned to several experimental groups with each group having three rats. The sham group received a carotid artery injury only. In the saline group, $100 \mu \mathrm{l}$ saline was injected into the tail vein after carotid artery injury. In the EPC group, $2 \times 10^{6}$ EPCs in $100 \mu \mathrm{l}$ saline were injected after carotid artery injury. In the VEGF-pretreated EPC group, $2 \times 10^{6}$ EPCs were preincubated with $50 \mathrm{ng} / \mathrm{mL}$ VEGF for $24 \mathrm{~h}$ and then injected into the tail vein after carotid artery injury. In the VEGF-pretreated EPC + siCx43 group, $2 \times 10^{6} \mathrm{EPCs}$ were incubated with $8 \mu \mathrm{lsiCx} 43$ for $6 \mathrm{~h}$ and $50 \mathrm{ng} / \mathrm{mL}$ VEGF for $24 \mathrm{~h}$ and then injected into the tail vein after carotid artery injury.

Carotid artery injury was performed as previously described [19]. Briefly, rats were anesthetized with pentobarbital sodium at a dose of $40 \mathrm{mg} / \mathrm{kg}$. The left carotid artery was exposed through a midline incision in the neck. Several silk sutures were applied to temporarily restrict blood flow to surgical areas. Then, a 1.5-F Fogarty balloon catheter (Baxter, Deerfield, IL, USA) was inserted through the external carotid artery, inflated, and passed three times along the segment. After the external carotid artery was permanently ligated, blood flow to the internal carotid artery was restored. The skin was closed with a single suture using 6-0 silk. Carotid arteries were harvested 7 or 28 days after carotid artery injury. Intimal hyperplasia was observed through hematoxylin and eosin (H\&E; Solarbio) staining using an inverted microscope.

\section{EPC differentiation after transplantation}

Before transplantation, EPCs $\left(2 \times 10^{6}\right)$ were incubated with $2.4 \mathrm{~g} / \mathrm{mL}$ DiI-ac-LDL for $1 \mathrm{~h}$. Then, the cells were washed with PBS and injected into the rat tail vein in $100 \mu \mathrm{l}$ saline after carotid artery injury. Carotid arteries were harvested 3 days after injury. After perfusion fixation with $4 \%$ PFA, the vessels were washed with PBS and embedded in optimal cutting temperature (OCT; Sakura, Torrance, CA, USA) compound. Subsequently, frozen sections of the carotid artery were prepared and incubated with FITC-UEA-I.

\section{Assessment of reendothelialization}

Assessment of reendothelialization was performed as previously described [17]. One week after carotid artery injury, $200 \mu \mathrm{l} \mathrm{5 \%}$ Evans blue (Sigma-Aldrich) was injected into the heart. Injured vessels were harvested 15 min later and then fixed in 4\% PFA (Sigma-Aldrich). The reendothelialized area was defined as the area that was not stained with Evans blue dye. Morphometric analysis of the reendothelialization rate was performed using Image-Pro Plus 5.1 (Media Cybernetics Inc., Rockville, MD, USA).

\section{Assessment of intimal hyperplasia}

Assessment of intimal hyperplasia was conducted as previously described [17]. Two weeks after carotid artery injury, injured vessels were harvested. The vessels were fixed in $4 \%$ PFA and embedded in OCT compound; thereafter, carotid cross-sections were prepared for H\&E staining. All sections were examined under an inverted microscope (Leica). Morphometric analysis of the intimal/medial area was performed using Image-Pro Plus 5.1 (Media Cybernetics Inc.).

\section{Statistical analysis}

All values are expressed as the mean \pm standard deviation (SD). Comparisons between multiple groups were tested by one-way analysis of variance (ANOVA) followed by Fisher's least significant difference (LSD) test. Statistical analyses were performed using SPSS v19.0 (IBM, Armonk, NY, USA). A value of $p<0.05$ was considered statistically significant. 


\section{Results}

Characterization of spleen-derived EPCs

Isolated spleen-derived MNCs exhibited a spindleshaped morphology after 4-7 days of culture (see Additional file 1). Most cells $(92.00 \pm 2.23 \%)$ were positive for both DiI-ac-LDL and FITC-UEA-I and were therefore identified as EPCs (Fig. 1a-d). Furthermore, the percentages of cells positive for CD34 (stem cell marker) and VEGFR2 (EC marker) were $67.77 \pm 3.84 \%$ and $73.80 \pm 2.65 \%$, respectively $\quad(n=3$; Fig. $1 \mathrm{e}$ and $\mathrm{f}$ ).

\section{VEGF promotes the expression of Cx43 in EPCs}

Immunofluorescence analysis indicated that $\mathrm{Cx} 43$ was located in both the cytomembrane and cytoplasm of EPCs (green in Fig. 2a). Control cells without primary antibody showed no staining (see Additional file 2). With increasing concentrations of VEGF, we observed a clear increase in relative $C x 43$ mRNA expression in EPCs $(1.78 \pm 0.25$ vs. $1.00 \pm 0.33$ with 50 and $0 \mathrm{ng} / \mathrm{mL}$ VEGF, respectively; $p<0.05$; Fig. $2 \mathrm{~b}$ ). In addition, VEGF significantly augmented relative $\mathrm{Cx} 43$ protein expression in EPCs $(2.07 \pm 0.49$ vs. $1.42 \pm 0.47$ vs. $1.00 \pm 0.31$ with 50 , 10 , and $0 \mathrm{ng} / \mathrm{mL}$ VEGF, respectively; $p<0.05$; Fig. $2 \mathrm{c}$ ).

\section{Transfection efficiency of Cx43 siRNA}

Western blot analysis was conducted to verify the transfection efficiency of Cx43 siRNA. After transfection for $48 \mathrm{~h}$, levels of $\mathrm{Cx} 43$ protein in the siCx43 group were considerably decreased compared to the negative control group (see Additional file 3).

\section{VEGF promotes Cx43-mediated GJ intercellular communication between adjacent EPCs}

FRAP was used to detect functional GJ intercellular communication (GJIC) between adjacent EPCs. The fluorescence recovery rate was higher in adjacent EPCs than in isolated EPCs, with significant differences between adjacent and isolated cells in the control group $(25.85 \pm 4.00 \%$ vs. $11.33 \pm 1.39 \% ; p<0.01)$, adjacent and isolated cells in the $\mathrm{EPC}+\mathrm{siCx} 43$ group $(18.52 \pm 3.38 \%$ vs. $10.43 \pm 1.23 \% ; p<0.01$ ), adjacent and isolated cells in the EPC + VEGF group $(65.65 \pm 6.24 \%$ vs. $14.57 \pm 1.16 \%$; $p<0.01)$, and adjacent and isolated cells in the EPC+ VEGF + siCx43 group $(31.31 \pm 1.53 \%$ vs. $14.83 \pm 7.48 \%$; $p<0.01)$. Transfection with Cx43 siRNA decreased the fluorescence recovery of bleached cells (18.52 $\pm 3.38 \%$ vs. $25.85 \pm 4.00 \%$ in the $\mathrm{EPC}+\mathrm{siCx} 43$ and control groups, respectively; $p<0.01)$. Administration of VEGF increased the fluorescence recovery of bleached cells
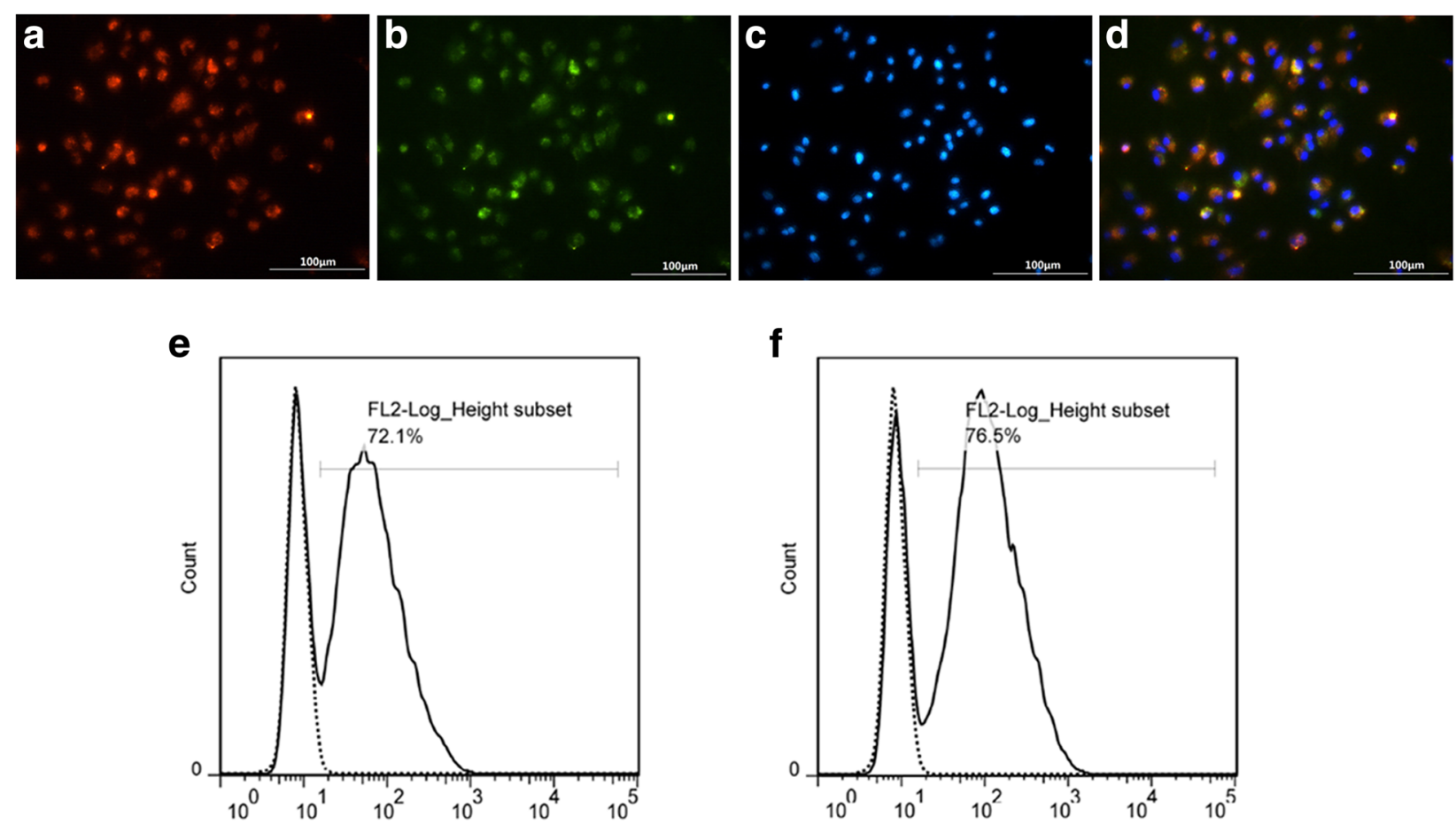

Fig. 1 Spleen-derived mononuclear cells (MNCs) differentiate into cells with the characteristics of endothelial progenitor cells (EPCs) in vitro. a-d Cells double-labeled for Dil-labeled acetylated low-density lipoprotein (Dil-ac-LDL; red) and fluorescein isothiocyanate (FITC)-lectin binding (green) were identified as EPCs (yellow). Cell nuclei were stained with DAPI (blue). e, f Adherent MNCs were analyzed for expression of CD34 and vascular endothelial growth factor receptor 2 (VEGFR2), respectively, by fluorescence-activated cell sorting (FACS). Dotted histograms represent isotype controls. Representative images from at least three experiments are shown. Scale bar $=100 \mu \mathrm{m}$ 


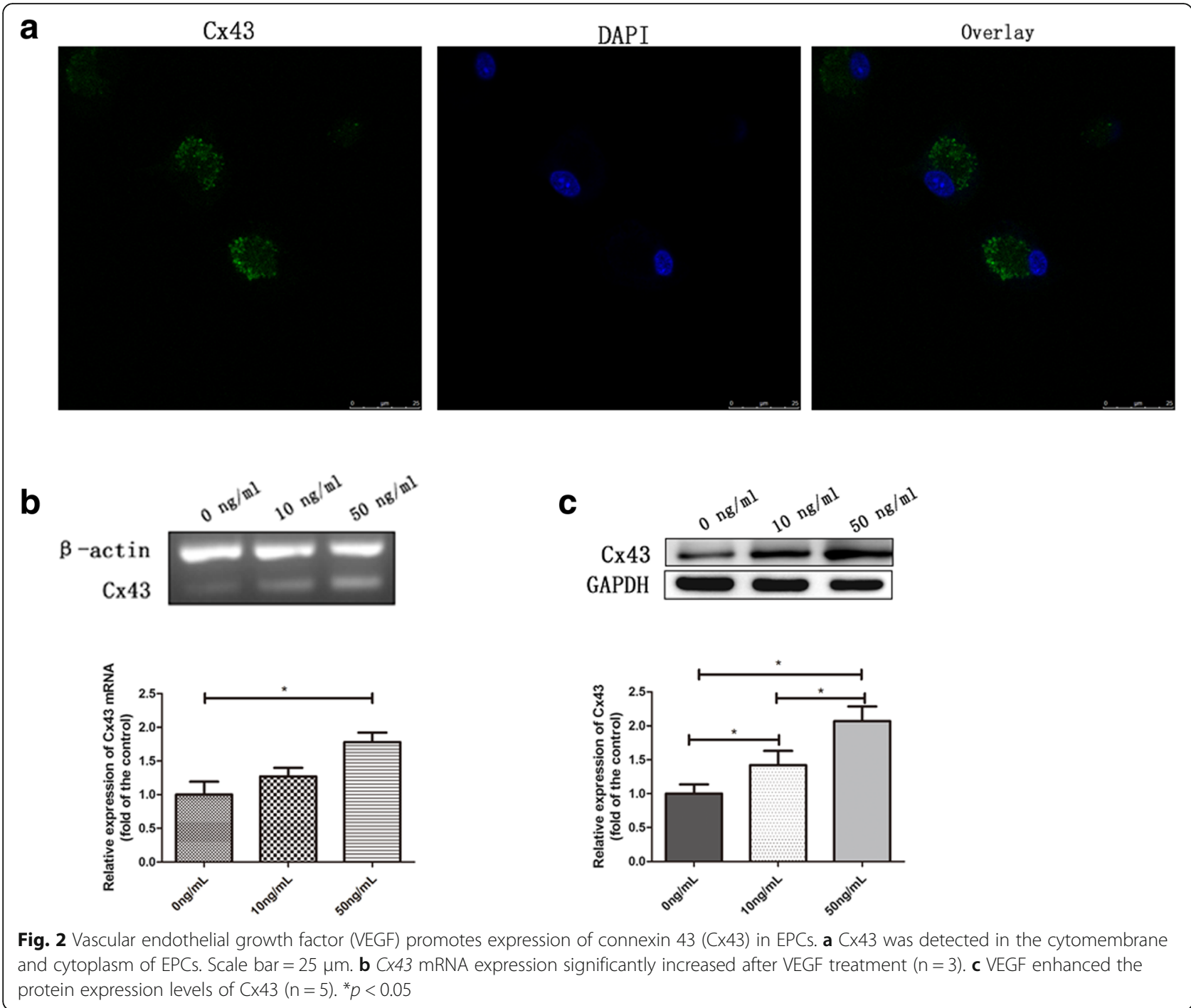

$(65.65 \pm 6.24 \%$ vs. $25.85 \pm 4.00 \%$ in the EPC + VEGF and control groups, respectively; $p<0.01$ ), whereas pretreatment with $\mathrm{Cx} 43$ siRNA reduced fluorescence recovery $(31.31 \pm 1.53 \%$ vs. $65.65 \pm 6.24 \%$ in the EPC + VEGF + siCx43 and EPC + VEGF groups, respectively; $p<0.01$; Fig. 3a).

\section{VEGF promotes Cx43-mediated differentiation of EPCs}

The differentiation of EPCs is characterized by the expression of EC markers (CD31 and vWF). To assess the effect of VEGF on Cx43-mediated EPC differentiation, EPCs were cultured with VEGF and/or Cx43 siRNA. Transfection with Cx43 siRNA blocked the relative protein expression of CD31 ( $0.79 \pm 0.05$ vs. $1.00 \pm 0.03$ in the $\mathrm{EPC}+\mathrm{siCx} 43$ and control groups, respectively; $p<0.01$ ) and vWF $(0.75 \pm 0.08$ vs. $1.00 \pm 0.08$ in the $\mathrm{EPC}+\mathrm{siCx} 43$ and control groups, respectively; $p<0.05$ ). EPCs cultured with VEGF displayed higher relative protein expression of
CD31 $(1.29 \pm 0.05$ vs. $1.00 \pm 0.03 ; p<0.001)$ and vWF $(1.35 \pm 0.13$ vs. $1.00 \pm 0.08 ; p<0.01)$ than control EPCs. However, silencing of $\mathrm{Cx} 43$ attenuated the relative protein expression of CD31 $(1.15 \pm 0.07$ vs. $1.29 \pm 0.05 ; p<0.01)$ and vWF $(1.09 \pm 0.11$ vs. $1.35 \pm 0.13 ; p<0.05)$ in EPCs treated with VEGF (Fig. 3b).

\section{VEGF pretreatment increases EPC homing via Cx43}

H\&E staining showed obvious neointima on days 7 and 28, confirming carotid artery injury (Fig. 4a). To determine whether EPCs were incorporated into the injured vessel walls and differentiated into ECs, animals received $2 \times 10^{6}$ DiI-labeled EPCs via tail vein injection. DiI-labeled EPCs, identified as red fluorescent cells, were seen lining the lumen via co-staining for the endothelial marker FITC-UEA. No DiI-labeled cells were observed in the saline group (see Additional file 4). Although differences were found between the EPC+ 


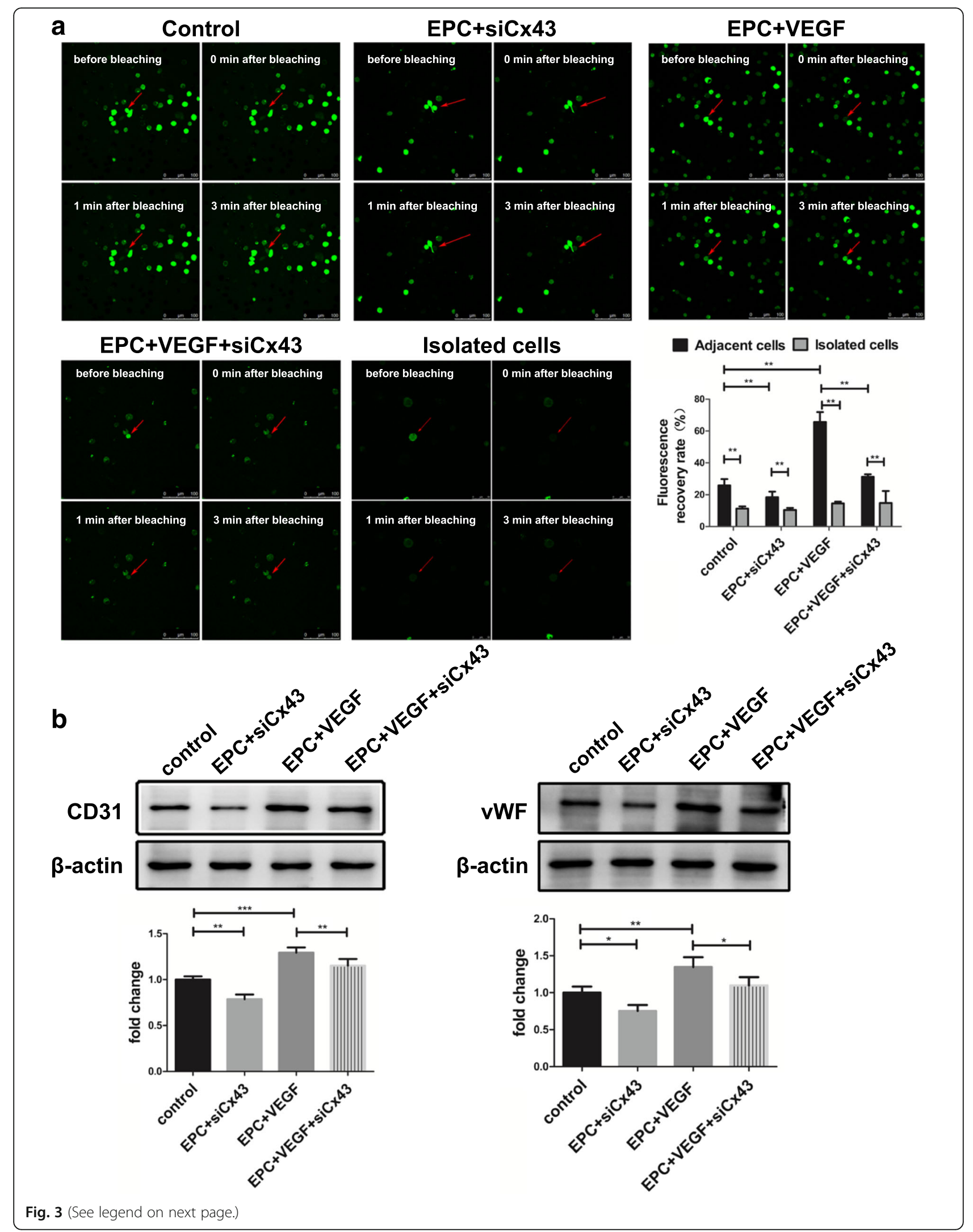


(See figure on previous page.)

Fig. 3 The role of Cx43-mediated gap junction intercellular communication (GJIC) in VEGF-induced EPC differentiation. a VEGF induced a significant increase in GJIC, while the silencing of Cx43 decreased GJIC $(n=6)$. The red arrow represents bleached cells. Scale bar $=100 \mu \mathrm{m}$. $\mathbf{b}$ VEGF promoted the expression of CD31 and von Willebrand factor (vWF). However, the silencing of Cx43 reduced the expression of CD31 and vWF ( $n=3$ ). ${ }^{*} p<0.05 ;{ }^{* *} p<0.01 ;{ }^{* * *} p<0.001$

siCx43 and EPC groups ( $3 \pm 1$ vs. $5.00 \pm 2.00)$, this difference was not statistically significant $(p=0.22)$. There was a significant increase in the number of EPCs in the injured areas after VEGF pretreatment $(15.67 \pm 2.08$ vs. $5.00 \pm$ 2.00 in the EPC + VEGF and EPC groups, respectively; $p<$ $0.01)$, which was significantly inhibited by $C x 43$ interference $(10.67 \pm 2.08$ vs. $15.67 \pm 2.08$ in the EPC $+\mathrm{VEGF}+$ siCx43 and EPC + VEGF groups, respectively; $p<0.05$; Fig. $4 \mathrm{~b}$ and $\mathrm{c})$.

\section{VEGF pretreatment before EPC transplantation promotes reendothelialization via $\mathrm{Cx} 43$}

To determine whether VEGF promoted endothelium recovery via $\mathrm{Cx} 43$, rats were injected with Evans blue 7 days after carotid artery injury. Reendothelialization was represented by the ratio of the reendothelialized area (white) to the total area (white + blue). Reendothelialization significantly increased after EPC transplantation (18.39 $\pm 1.83 \%$ vs. $5.51 \pm 1.18 \%$ in the EPC and saline groups, respectively; $p<0.01)$. Transfection with Cx43 siRNA inhibited reendothelialization $(13.73 \pm 0.575 \%$ vs. $18.39 \pm 1.83 \%$ in the EPC + siCx43 and EPC groups, respectively; $p<0.01)$. This effect was strengthened by VEGF pretreatment (52.66 \pm $3.78 \%$ vs. $18.39 \pm 1.83 \%$ in the EPC + VEGF and EPC groups, respectively; $p<0.01)$. However, the silencing of Cx43 attenuated reendothelialization $(29.58 \pm 2.44 \%$ vs. $52.66 \pm 3.78 \%$ in the EPC + VEGF + siCx43 and EPC + VEGF groups, respectively; $p<0.01$; Fig. $5 \mathrm{a}$ and b).

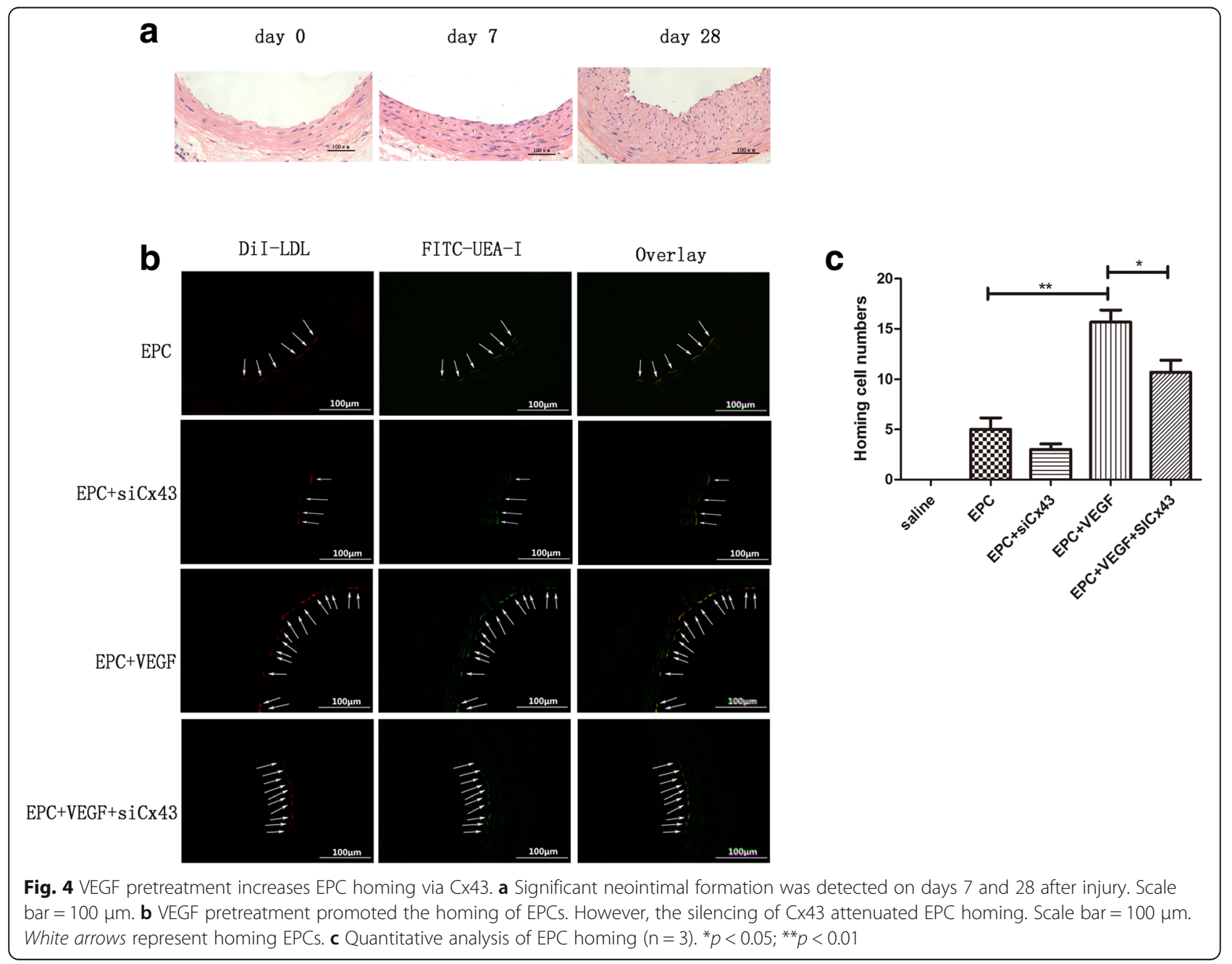




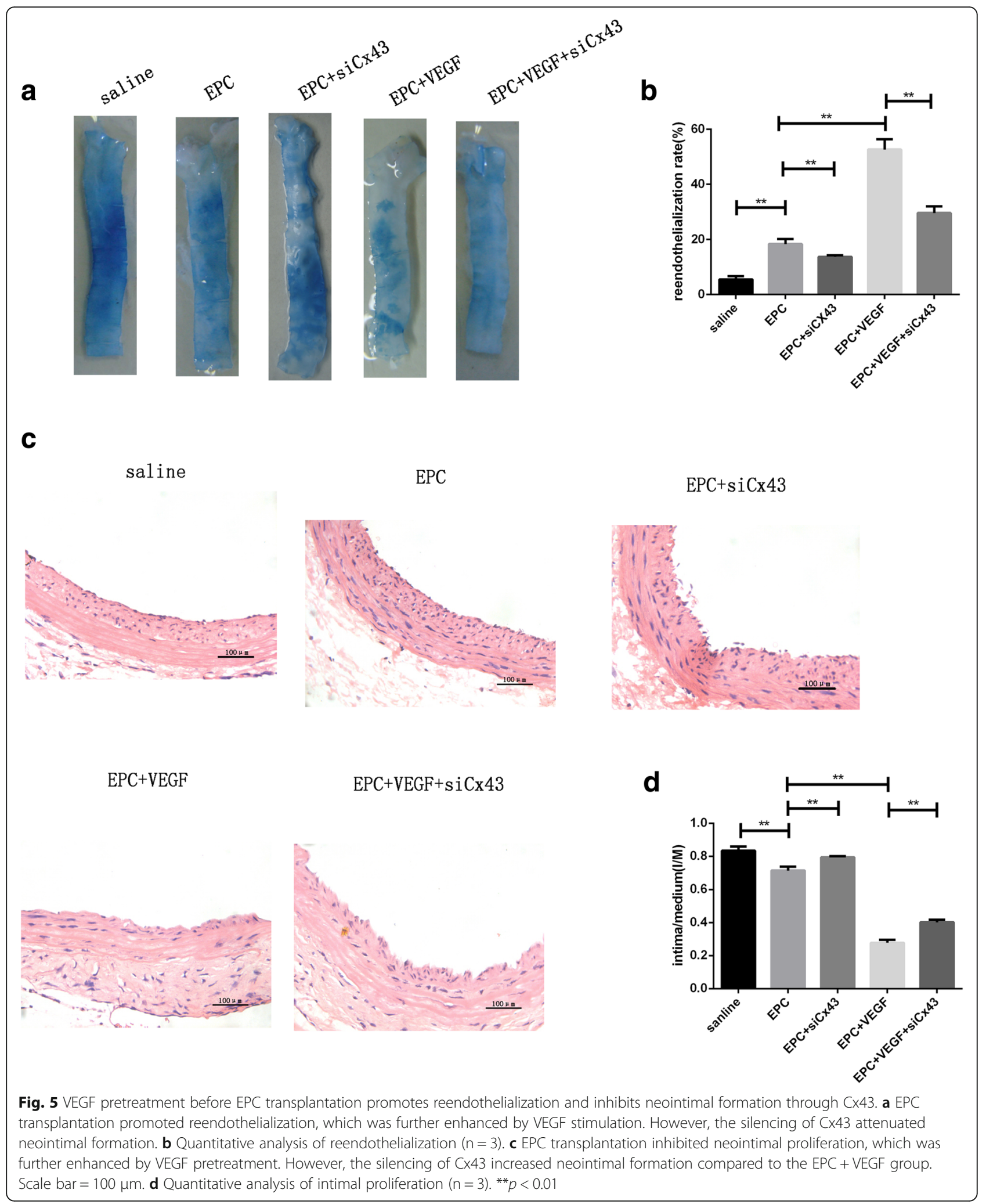




\section{VEGF pretreatment before EPC transplantation inhibits} neointima formation via $\mathrm{Cx} 43$

The transplantation of EPCs significantly inhibited neointimal development in injured vessels, as determined by the ratio of the intimal to medial areas $(0.714 \pm 0.024$ vs. $0.835 \pm 0.025$ in the EPC and saline groups, respectively; $p<0.01)$. Transfection with Cx43 siRNA impaired the ability of EPC inhibition of intimal proliferation (0.794 \pm 0.065 vs. $0.714 \pm 0.024$ in the $\mathrm{EPC}+\mathrm{siCx} 43$ and $\mathrm{EPC}$ groups, respectively; $p<0.01$ ). VEGF pretreatment further inhibit neointima formation $(0.278 \pm 0.018$ vs. $0.714 \pm 0.024$ in the EPC $+\mathrm{VEGF}$ and EPC groups, respectively; $p<0.01)$. However, $\mathrm{Cx} 43$ silencing increased intimal hyperplasia $(0.402 \pm 0.116$ vs. $0.278 \pm$ 0.018 in the EPC + VEGF + siCx43 and EPC + VEGF groups, respectively; $p<0.01$; Fig. $5 c$ and d).

\section{Discussion}

Our results from in vivo and in vitro experiments indicated that VEGF promotes EPC differentiation and vascular repair through $\mathrm{Cx} 43$.

\section{EPCs promotes endothelial and vascular repair}

The endothelium serves as a barrier between vessel wall and blood, and plays an essential role in regulating vascular homeostasis. Promoting endothelial generation is a therapeutic option for vascular repair; it has been demonstrated that EPCs-derived from hematopoietic stem cells-can mobilize into peripheral blood, home to an injured zone, differentiate into ECs, and promote vascular repair $[4,20]$.

\section{VEGF is a crucial regulator in vascular injury repair}

VEGF has been shown to induce angiogenesis, accelerating endothelial repair and preventing intimal hyperplasia after vascular injury in vitro and in vivo [21-24]. One study found that EPCs transfected with adenovirus encoding for VEGF are more capable of homing to denuded areas and differentiating into ECs [25]. In our study, we also found that VEGF promotes EPC differentiation, homing, and repair of injured blood vessels.

Mechanism of VEGF-induced EPC differentiation is unclear It has been reported that VEGF induces EPC differentiation in in vivo and in vitro experiments, and therefore contributes to vascular repair [25-27]. Several mechanisms are suggested to be involved in this process. Some reports indicate that VEGF appears to regulate EPC differentiation through PI3K/Akt/eNOS signaling [28, 29]; one report suggests that the Akt/HDAC3/p53 pathway is involved [30]; and the serine/threonine-protein kinase Pim-1 [7, 27], as well as the MAPK/ERK signaling pathway $[8,31]$, also appear to participate. However, the specific mechanisms of VEGF-induced EPC differentiation and blood vessel repair remain unclear.

\section{Cx43 potentially modulates EPC differentiation}

Of the 21 known connexins, vascular endothelial cells primarily express three: Cx37, Cx40, and Cx43 [18], all of which facilitate the propagation of electrical and chemical signals along the vessel wall. GJs in EPCs mainly consist of $\mathrm{Cx} 43$, and EPCs treated with $\mathrm{Cx} 43$ siRNA lose their therapeutic potential [14]. These findings link the adjustment of Cx43-mediated EPC function to the initiation and promotion of atherosclerosis. There is accumulating evidence that $\mathrm{Cx} 43$ is capable of inducing GJ formation between stem cells or between stem cells and mature differentiated cells, and that downregulation or upregulation of $\mathrm{Cx} 43$ can suppress or boost stem cell differentiation, respectively $[11,12,32,33]$. It has also been demonstrated that Cx43-mediated GJs participate in EPC proliferation, migration, and angiogenesis [14]. Therefore, we aimed to directly test whether CX43 participates in EPC differentiation. Our study shows that VEGF promotes EPC differentiation and vascular repair through Cx43-mediated GJs.

\section{Possible mechanisms for Cx43 induction of EPC differentiation}

Regarding the underlying mechanisms by which $\mathrm{Cx} 43$ induces EPC differentiation, cAMP/PKA and IP3/Ca2+ are proposed to be involved. One study found that cyclic adenosine monophosphate (cAMP) enhanced VEGFinduced EPC differentiation through activation of protein kinase A (PKA) signaling, and that blockade or upregulation of PKA expression reduced or enhanced the effect, respectively [34, 35]. These findings demonstrate that GJ-mediated exchange of cAMP could modulate EPC differentiation through PKA signaling.

$\mathrm{Ca}^{2+}$ is an important second messenger in the regulation of cell physiological functions; and inositol triphosphate 3 (IP3) may open calcium channels in the endoplasmic reticulum and increase intracellular calcium concentration. The distribution and transfer of $\mathrm{Ca}^{2+}$ through GJs may activate specific proteins, or may combine with specific enzymes, modulating cellular functions. Some studies have suggested that VEGF may activate phospholipase C (PLC- $\gamma$ ), hydrolyze phosphatidylinositol-4,5-bisphosphate (PIP2), increase cellular calcium concentration, regulate the expression of endothelial differentiationrelated genes, and promote EPC differentiation [36, 37]. Therefore, the Cx43-mediated exchange of intercellular second messengers such as $\mathrm{Ca}^{2+}$ is clearly a potential mechanism by which $\mathrm{Cx} 43$ regulates EPC differentiation. 


\section{Exploration of potential therapeutic applications}

Recent efforts have been made to apply the differentiationpromoting capabilities of VEGF and Cx43 to clinical research settings. Several studies report that EPCs captured on VEGF-bound stents display rapid differentiation $[38,39]$, and $C x 43$ has been demonstrated to have an important role in maintaining the normal physiological functions of EPCs and ECs [14, 40, 41]. The injection of targeting nanoparticles loaded with Cx43-overexpressing adenoviruses, combined with EPCs, might be an effective method of treating vascular injuries and vascular pathologies. Alternatively, using stents to load Cx43-overexpressing EPCs into blood vessels is another possible therapeutic treatment for vascular injuries and diseases.

\section{Limitations}

We believe that cell density plays a role in $\mathrm{Cx} 43$ mediated, VEGF-induced EPC differentiation. Our decisions to use cell densities of $1 \times 10^{6} \mathrm{cells} / \mathrm{cm}^{2}$ for our in vitro experiments and $2 \times 10^{6}$ cells $/ 100 \mu \mathrm{L}$ for our in vivo experiments were based on methods described in previous studies [19], but we hope to elucidate the specific influence of cell density in the future. Although we were able to demonstrate that VEGF promoted EPC differentiation through Cx43-induced GJs, we were unable to explore additional mechanisms of EPC differentiation because of limited experimental conditions. We hope to conduct future experiments that will employ microfabrication and laser-guided cell micropatterning techniques [33], as well as dual patch clamp recording techniques [42].

\section{Conclusions}

In summary, we report that VEGF, at least in part, promotes EPC differentiation and vascular repair via Cx43. Our findings indicate that $\mathrm{Cx} 43$ is a potential therapeutic target for blood vessel injury diseases.

\section{Additional files}

Additional file 1: Morphology of mononuclear cells (MNCs). (A) Smaller cells were diffusely distributed in culture solution after 1 day. (B) Cells became larger after culture for 4 days. (C) Cells exhibited a spindle-shaped, endothelial cell-like morphology after culture for 7 days. (TIF $1954 \mathrm{~kb}$ )

Additional file 2: Nonspecific fluorescence detection of endothelial progenitor cells (EPCs). EPCs were incubated with saline instead of anti-Cx43 primary antibody, and nuclei were stained with DAPI. Scale bar $=25 \mu \mathrm{m}$. (TIF $736 \mathrm{~kb}$ )

Additional file 3: Efficiency of connexin 43 (Cx43) short interfering RNA (siRNA). Treatment with $\mathrm{C} \times 43$ siRNA effectively reduced $\mathrm{C} \times 43$ protein expression $(n=3) .{ }^{*} p<0.05$. NC negative control. (TIF $242 \mathrm{~kb}$ )

Additional file 4: Fluorescent tracer technique employed to detect saline group homing. No EPCs were observed in the area of the injured vessel. (TIF $2403 \mathrm{~kb}$ )

\begin{abstract}
Abbreviations
ANOVA: Analysis of variance; BSA: Bovine serum albumin; Cx43: connexin 43; DAPI: 4,6-diamidino-2-phenylindole; Dil-ac-LDL: Dil-labeled acetylated lowdensity lipoprotein; DMEM: Dulbecco's modified Eagle's medium;

EC: Endothelial cell; ECL: Enhanced chemiluminescence; EPC: Endothelial progenitor cell; FACS: Fluorescence-activated cell sorting; FITC-UEA-

I: Fluorescein isothiocyanate (FITC)-labeled Ulex europaeus agglutinin I; FRAP: Fluorescence redistribution after photobleaching;

GAPDH: Glyceraldehyde-3-phosphate dehydrogenase; GJ: Gap junction;

GJIC: Gap junction intercellular communication; H\&E: Hematoxylin and eosin; LSCM: Laser scanning confocal microscopy; LSD: Least significant difference; MAPK/ERK: Mitogen-activated protein kinase/extracellular signal-related kinase; MNC: Mononuclear cell; OCT: Optimal cutting temperature; PBS: Phosphate-buffered saline; PE: Phycoerythrin; PFA: Paraformaldehyde; PKA: Protein kinase A; PMSF: Phenylmethane sulfonyl fluoride;

PVDF: Polyvinylidene fluoride; RIPA: Radioimmunoprecipitation assay; RT: Reverse transcriptase; SD: Standard deviation; siRNA: Short interfering RNA; VEGF: Vascular endothelial growth factor; VEGFR: Vascular endothelial growth factor receptor; vWF: von Willebrand factor
\end{abstract}

\section{Acknowledgements}

The authors would like to thank Editage for providing English language editing.

\section{Funding}

This study was supported by grants from the National Natural Science Foundation of China (81370213 and 81670428).

\section{Availability of data and materials}

All data supporting the findings of this study are available within the article and its additional information files.

\section{Authors' contributions}

XZ supervised the project. LL and XZ conceived the project and designed the experiments. $L L$ performed the majority of the experiments. $H L$ assisted with RT-PCR analysis. CX and MD conducted parts of the cell culture experiments and the western blotting analysis. MD carried out the carotid artery injury and identification process. MS helped with FRAP experiments. $X Y$ and $S X$ provided technical advice. $L L$ and $X Z$ interpreted the data and wrote the manuscript, with comments from the rest of the authors. SX and XZ helped to revise the manuscript. All authors read and approved the final manuscript.

\section{Ethics approval}

All procedures were conducted with the approval of the ethics committee of the Third Military Medical University and in accordance with the National Institutes of Health Guidelines for the Care and Use of Laboratory Animals (8th edition, National Research Council, USA, 2011).

Consent for publication

Not applicable.

Competing interests

The authors declare that they have no competing interests.

\section{Publisher's Note}

Springer Nature remains neutral with regard to jurisdictional claims in published maps and institutional affiliations.

\section{Author details}

'Institute of Cardiovascular Research, Xinqiao Hospital, Third Military Medical University, Chongqing 400037, China. ${ }^{2}$ Cardiovascular Department, First People's Hospital of Chong Qing Liang Jiang New Zone, Chongqing 401120, China. ${ }^{3}$ Department of Occupational Health, Third Military Medical University, Chongqing 400038, China. 
Received: 14 July 2017 Revised: 27 September 2017 Accepted: 28 September 2017 Published online: 24 October 2017

\section{References}

1. Poredos $P$, Jezovnik MK. Testing endothelial function and its clinical relevance. J Atheroscler Thromb. 2013;20:1-8.

2. Deanfield JE, Halcox JP, Rabelink TJ. Endothelial function and dysfunction: testing and clinical relevance. Circulation. 2007;115:1285-95.

3. Yin Y, Liu H, Wang F, et al. Transplantation of cryopreserved human umbilical cord blood-derived endothelial progenitor cells induces recovery of carotid artery injury in nude rats. Stem Cell Res Ther. 2015;6:37.

4. Yin $Y$, Zhao $X$, Fang $Y$, et al. SDF-1alpha involved in mobilization and recruitment of endothelial progenitor cells after arterial injury in mice. Cardiovasc Pathol. 2010;19:218-27.

5. Kalka C, Masuda H, Takahashi T, et al. Vascular endothelial growth factor(165) gene transfer augments circulating endothelial progenitor cells in human subjects. Circ Res. 2000;86:1198-202.

6. Young PP, Hofling AA, Sands MS. VEGF increases engraftment of bone marrow-derived endothelial progenitor cells (EPCS) into vasculature of newborn murine recipients. Proc Natl Acad Sci U S A. 2002;99:11951-6.

7. Kawasaki K, Watabe T, Sase H, et al. Ras signaling directs endothelial specification of VEGFR2+ vascular progenitor cells. J Cell Biol. 2008;181:131-41.

8. $\mathrm{Xu}$ J, Liu $X$, Jiang $Y$, et al. MAPKJERK signalling mediates VEGF-induced bone marrow stem cell differentiation into endothelial cell. J Cell Mol Med. 2008; 12:2395-406.

9. Decrock $\mathrm{E}$, Vinken $\mathrm{M}, \mathrm{Bol} \mathrm{M}$, et al. Calcium and connexin-based intercellular communication, a deadly catch? Cell Calcium. 2011;50:310-21.

10. Pfenniger A, Chanson M, Kwak BR. Connexins in atherosclerosis. Biochim Biophys Acta. 1828;2013:157-66.

11. Ke Q, Li L, Cai B, et al. Connexin 43 is involved in the generation of humaninduced pluripotent stem cells. Hum Mol Genet. 2013;22:2221-33.

12. Ramkisoensing A, Pijnappels $D$, Schalij $M$, et al. Connexin43 expression is essential for functional cardiomyogenic differentiation of human fetal mesenchymal stem cells. J Am Coll Cardiol. 2012;59S:E855.

13. Song M, Yu X, Cui X, et al. Blockade of connexin 43 hemichannels reduces neointima formation after vascular injury by inhibiting proliferation and phenotypic modulation of smooth muscle cells. Exp Biol Med (Maywood). 2009;234:1192-200.

14. Wang HH, Su CH, Wu YJ, et al. Reduction of connexin43 in human endothelial progenitor cells impairs the angiogenic potential. Angiogenesis. 2013;16:553-60.

15. Behrens J, Kameritsch P, Wallner S, et al. The carboxyl tail of Cx43 augments p38 mediated cell migration in a gap junction-independent manner. Eur J Cell Biol. 2010;89:828-38.

16. Zhao $X$, Huang $L$, Yin $Y$, et al. Estrogen induces endothelial progenitor cells proliferation and migration by estrogen receptors and PI3K-dependent pathways. Microvasc Res. 2008;75:45-52.

17. Zhao X, Huang L, Yin Y, et al. Autologous endothelial progenitor cells transplantation promoting endothelial recovery in mice. Transpl Int. 2007;20: 712-21.

18. Brisset AC, Isakson BE, Kwak BR. Connexins in vascular physiology and pathology. Antioxid Redox Signal. 2009;11:267-82.

19. Cui B, Huang L, Fang $Y$, et al. Transplantation of endothelial progenitor cells overexpressing endothelial nitric oxide synthase enhances inhibition of neointimal hyperplasia and restores endothelium-dependent vasodilatation. Microvasc Res. 2011:81:143-50.

20. Yin $Y$, Huang $L$, Zhao $X$, et al. AMD3100 mobilizes endothelial progenitor cells in mice, but inhibits its biological functions by blocking an autocrine/ paracrine regulatory loop of stromal cell derived factor-1 in vitro. J Cardiovasc Pharmacol. 2007;50:61-7.

21. Chen J, Gu Z, Wu M, et al. C-reactive protein can upregulate VEGF expression to promote ADSC-induced angiogenesis by activating HIF-1a via CD64/PI3K/ Akt and MAPKJERK signaling pathways. Stem Cell Res Ther. 2016;7:114

22. Tratwal J, Mathiasen AB, Juhl M, et al. Influence of vascular endothelial growth factor stimulation and serum deprivation on gene activation patterns of human adipose tissue-derived stromal cells. Stem Cell Res Ther. 2015;6:62

23. Hutter R. Vascular endothelial growth factor regulates reendothelialization and neointima formation in a mouse model of arterial injury. Circulation. 2004; 110:2430-5
24. Asahara T, Takahashi T, Masuda H, et al. VEGF contributes to postnatal neovascularization by mobilizing bone marrow-derived endothelial progenitor cells. EMBO J. 1999;18:3964-72.

25. Iwaguro $\mathrm{H}$, Yamaguchi J, Kalka $\mathrm{C}$, et al. Endothelial progenitor cell vascular endothelial growth factor gene transfer for vascular regeneration. Circulation. 2002;105:732-8.

26. Matsuda T, Kuwana M, Aomizu T, et al. Surface design for in situ capture of endothelial progenitor cells: VEGF-bound surface architecture and behaviors of cultured mononuclear cells. J Biomed Mater Res B Appl Biomater. 2013; 101:50-60.

27. Urbich C. Endothelial progenitor cells: characterization and role in vascular biology. Circ Res. 2004;95:343-53.

28. Imanishi T, Hano T, Matsuo Y, et al. Oxidized low-density lipoprotein inhibits vascular endothelial growth factor-induced endothelial progenitor cell differentiation. Clin Exp Pharmacol Physiol. 2003:30:665-70.

29. Kim J, Jung S, Kwon Y, et al. Ginsenoside Rg3 inhibits endothelial progenitor cell differentiation through attenuation of VEGF-dependent Akt/eNOS signaling. Phytother Res. 2012;26:1286-93.

30. Zeng L, Xiao Q, Margariti A, et al. HDAC3 is crucial in shear- and VEGFinduced stem cell differentiation toward endothelial cells. J Cell Biol. 2006; 174:1059-69.

31. Almalki SG, Agrawal DK. ERK signaling is required for VEGF-ANEGFR2induced differentiation of porcine adipose-derived mesenchymal stem cells into endothelial cells. Stem Cell Res Ther. 2017:8:113.

32. Carvalho $\mathrm{J}$, Braga VBA, Melo MB, et al. Priming mesenchymal stem cells boosts stem cell therapy to treat myocardial infarction. J Cell Mol Med. 2013;17:617-25.

33. Ma Z, Liu Q, Liu H, et al. Cardiogenic regulation of stem-cell electrical properties in a laser-patterned biochip. Cell Mol Bioeng. 2012;5:327-36.

34. Yamamizu K, Kawasaki K, Katayama S, et al. Enhancement of vascular progenitor potential by protein kinase A through dual induction of Flk-1 and Neuropilin-1. Blood. 2009;114:3707-16.

35. Bleiziffer $\mathrm{O}$, Horch RE, Hammon $\mathrm{M}$, et al. T17b murine embryonal endothelial progenitor cells can be induced towards both proliferation and differentiation in a fibrin matrix. J Cell Mol Med. 2009:13:926-35.

36. Dragoni S, Laforenza U, Bonetti E, et al. Vascular endothelial growth factor stimulates endothelial colony forming cells proliferation and tubulogenesis by inducing oscillations in intracellular Ca2+ concentration. Stem Cells. 2011;29:1898-907.

37. Moccia F, Lodola F, Dragoni S, et al. Ca2+ signalling in endothelial progenitor cells: a novel means to improve cell-based therapy and impair tumour vascularisation. Curr Vasc Pharmacol. 2014:12:87-105.

38. Suzuki Y, Yamamoto K, Ando J, et al. Arterial shear stress augments the differentiation of endothelial progenitor cells adhered to VEGF-bound surfaces. Biochem Biophys Res Commun. 2012;423:91-7.

39. Song $C L$, Li Q, Zhang JC, et al. Study of a novel coating strategy for coronary stents: evaluation of stainless metallic steel coated with VEGF and anti-CD34 antibody in vitro. Eur Rev Med Pharmacol Sci. 2016;20:311-6.

40. Yeh HI, Lai YJ, Chang HM, et al. Multiple connexin expression in regenerating arterial endothelial gap junctions. Arterioscler Thromb Vasc Biol. 2000;20:1753-62.

41. Liao Y, Day KH, Damon DN, et al. Endothelial cell-specific knockout of connexin 43 causes hypotension and bradycardia in mice. Proc Natl Acad Sci U S A. 2001;98:9989-94

42. Veenstra RD. Establishment of the dual whole cell recording patch clamp configuration for the measurement of gap junction conductance. Methods Mol Biol. 2016:1437:213-31. 